mesothelioma (MM). This result suggest the decrease in stimulation-induced cytotoxicity in MM patients. Therefore, we hypothesised that chronic exposure to asbestos might affect anti-tumour immunity of CTL, and examined this possibility by comparing long-term cultures of human $\mathrm{CD}^{+} \mathrm{T}$ cell line EBT-8 between with and without exposure to chrysotile $(\mathrm{CH})$ asbestos as an in vitro model to analyse the effects of exposure on CTL.

Methods EBT-8 cells were continuously cultured with $\mathrm{CH}$ asbestos at 0,5 , or $30 \mu \mathrm{g} / \mathrm{ml}$, and were designated EBT-8Org, EBT-8-CH5, and EBT-8-CH30, respectively. The cells were regularly separated from asbestos using a Ficoll-Hypaque density gradient, before being assayed for MFI of granzyme B and percentage of perforin ${ }^{+}$cells by flow cytometry. The cells were also stimulated with PMA/ionomycin for 4 hour or beads coated with anti CD3 Ab for 48 hour and assayed for IFN- $\lambda$ production by flow cytometry and ELISA, respectively.

Result The long-term exposure to $\mathrm{CH}$ asbestos at $5 \mu \mathrm{g} / \mathrm{ml}$ or $30 \mu \mathrm{g} / \mathrm{ml}$ did not suppress the MFI of granzyme B in EBT-8 cells. In contrast, both the doses of $\mathrm{CH}$ exposure suppressed the percentage of perforin ${ }^{+}$cells. Although the exposure to $\mathrm{CH}$ did not suppress intracellular level of IFN- $\lambda$ induced by PMA/ionomycin, the secreted production of IFN- $\lambda$ stimulated via CD3 decreased by $\mathrm{CH}$ exposure.

Discussion These results indicate that long-term exposure to asbestos has the potential to suppress the perforin level and the production of IFN- $\lambda$ of human $\mathrm{CD}^{+} \mathrm{T}$ cells. Further study is needed to clarify the mechanism of asbestos-caused suppressed function of CTL.

\section{EFFECT OF ASBESTOS ON FOXP3 EXPRESSION IN HUMAN T CELL LINE MT-2}

${ }^{1} \mathrm{H}$ Matsuzaki*, 'S Lee, ${ }^{2} \mathrm{M}$ Maeda, ${ }^{1} \mathrm{~N}$ Kumagai-takei, ${ }^{1} \mathrm{~K}$ Yoshitome, ${ }^{1} \mathrm{Y}$ Nishimura, ${ }^{1} \mathrm{~T}$ Otsuki. 'Department of Hygiene, Kawasaki Medical School, Kurashiki, Japan; ${ }^{2}$ Okayama University Graduate School of Natural Science and Technology, Okayama, Japan

\subsection{6/oemed-2018-ICOHabstracts. 163}

Introduction Asbestos fibres cause mesothelioma and lung cancer. We propose that asbestos suppress anti-tumour immune system in addition to transformation of mesothelial and lung epithelial cells. It is considered that regulatory $\mathrm{T}$ cells, Treg produce inhibitory cytokines to suppress immune reaction against tumour cells. We employed human T cell line MT-2 cell as a model of Treg and cultured them with low concentration of asbestos for 8 months. MT-2 cells exposed with low-concentration asbestos for long term showed higher viability after treatment with high lethal dose of asbestos than original MT-2 cells, and they were designated as MT-2Rst. However, it is still unclear how asbestos induces apoptosis in MT-2 cells and molecular basis of resistance of MT-2Rst cells to high concentration of asbestos. Recently, we found that forkhead transcription factor FoxP3 plays an important role in regulation of apoptosis induced by asbestos.

Methods We analysed the regulation of FoxP3 transcription in MT-2Org and MT-2Rst cells using luciferase reporter plasmids containing promoter sequence of FoxP3 gene.

Result There was no significant difference in FoxP3 reporter activity between MT-2Org and MT-2Rst cells.
Discussion Our result indicates that long-term exposure with asbestos suppressed FoxP3 transcription through the epigenetic modification, such as DNA methylation.

\section{EFFECT OF SHORT-TERM EXPOSURE OF ASBESTOS ON HUMAN T CELL LINE MT-2}

${ }^{1} \mathrm{H}$ Matsuzaki*, ${ }^{1} \mathrm{~S}$ Lee, ${ }^{2} \mathrm{M}$ Maeda, ${ }^{1} \mathrm{~N}$ Kumagai-takei, ${ }^{1} \mathrm{~K}$ Yoshitome, ${ }^{1} \mathrm{Y}$ Nishimura, ${ }^{1} \mathrm{~T}$ Otsuki. 'Department of Hygiene, Kawasaki Medical School, Kurashiki, Japan; ${ }^{2}$ Okayama University Graduate School of Natural Science and Technology, Okayama, Japan

\subsection{6/oemed-2018-ICOHabstracts. 164}

Introduction Asbestos fibres cause mesothelioma and lung cancer. We propose that asbestos suppress anti-tumour immune system in addition to transformation of mesothelial and lung epithelial cells. It is reported that asbestos induces apoptosis of epitherial cells through various processes such as, mitochondrial dysfunction, DNA damage, ER stress. On the other hand, we proposed that asbestos fibres affects on immune cells to attenuate immune response to tumour cells. We employed MT-2 cells as a model of Treg and maintained them with low concentration of asbestos for longer than 8 months. MT-2 cells exposed with low-concentration asbestos for long term showed higher viability after treatment with high concentration of asbestos than original MT-2 cells, and they were designated as MT-2Rst. However, it is still unclear how asbestos induces apoptosis of MT-2 cells and molecular basis of resistance of MT-2Rst cells to high concentration of asbestos.

Methods We analysed acute effect of asbestos on mitochondria using mitochondrial membrane potential indicator JC-1 dye and immunoblot analysis using antibodies recognising DNA damage markers.

Result It was found that asbestos fibres induced loss of mitochondrial membrane potential and phosphorylation of Histone H2AX, a marker protein of DNA damage.

Discussion These data suggest that asbestos fibres induces apoptosis of MT-2 cells through the mitochondrial dysfunction and DNA damage, and these apoptotic pathways are modified in MT-2Rst cells.

\section{NANOTOXICITY OF TITANIUM NANOSHEETS FOR HUMAN IMMUNE CELLS}

${ }^{1} \mathrm{Y}$ Nishimura*, ${ }^{2} \mathrm{D}$ Yoshioka, ${ }^{1} \mathrm{~N}$ Kumagai-Takei, ${ }^{1} \mathrm{H}$ Matsuzaki, ${ }^{1} \mathrm{~S}$ Lee, ${ }^{1} \mathrm{~K}$ Yoshitome, ${ }^{1} \mathrm{~T}$ Otsuki. 'Department of Hygiene, Kawasaki Medical School, Kurashiki, Japan; ${ }^{2}$ Department of Natural Sciences, Kawasaki Medical School, Kurashiki, Japan

\subsection{6/oemed-2018-ICOHabstracts. 165}

Introduction The characteristic toxicity of nano-scaled materials, that is nanotoxicity, is a recent problem arising in association with nanotechnology. Titanium nanosheets (TNS) are known as 2D materials composed of titanium and oxygen with very thin structure and expected to be valuable for industrial usage. The present study examined the effect of exposure to TNS on human immune cells.

Methods Human peripheral blood mononuclear cells (PBMC) or magnetically isolated $\mathrm{CD} 14^{+}$monocyte or $\mathrm{CD}^{+} \mathrm{T}$ cells were cultured with TNS. Apoptosis was assayed by flow cytometry with annexin $\mathrm{V}$ staining. Intracellular 
microstructures of monocytes were observed by TEM and SEM, and titanium was identified by energy dispersion type X-ray spectroscopy (EDX). Adherent monocytes were pre-cultured with Alexa 568 dextran (A-Dex) to visualise endosomes. Results TNS exposure induced apoptosis of PBMC in the 7 days culture, the dose dependency of which was similar to asbestos, although apoptosis was not induced at the early stage of day 2 unlike asbestos. The apoptosis was inhibited by Q$\mathrm{VD}-\mathrm{OPh}$ pan-caspase inhibitor. Isolated $\mathrm{CD}^{+}{ }^{+} \mathrm{T}$ cells as well as monocytes showed apoptosis caused by TNS exposure, whereas monocytes showed giant vacuole formation prior to apoptosis. TNS-like compounds in vacuoles were observed by TEM, and SEM images showed rough surface of the inner layer of vacuolar membrane, in which titanium was identified by EDX. Most of vacuoles showed co-localization with fluorescence of A-Dex.

Conclusion These results indicate that TNS have toxic effect to cause caspase-dependent apoptosis of immune cells. In particular, TNS showed characteristic toxicity for monocytes, in which engulfed TNS were thought to enter into the endosomal pathway, leading to vacuole formation followed by apoptosis. Those findings suggest hazardous risk of occupational exposure to TNS.

\section{BIOLOGICAL EFFECTS OF CLOTH CONTAINING SPECIFIC ORE POWDER IN PATIENTS WITH POLLEN ALLERGY}

${ }^{1} \mathrm{~S}$ Lee*, ${ }^{2} \mathrm{H}$ Okamoto, ${ }^{1} \mathrm{~S}$ Yamamoto, ${ }^{1} \mathrm{~T}$ Hatayama, ${ }^{1} \mathrm{H}$ Matsuzaki, ${ }^{1} \mathrm{~N}$ Kumagai-Takei, ${ }^{1} \mathrm{~K}$ Yoshitome, ${ }^{1} \mathrm{Y}$ Nishimura, ${ }^{3} \mathrm{~T}$ Sato, ${ }^{4} \mathrm{Y}$ Kirita, ${ }^{2} \mathrm{Y}$ Fujii, ${ }^{1} \mathrm{~T}$ Otsuki. ${ }^{1}$ Dept. Hygiene, Kawasaki medical school, Kurashiki, Japan; ${ }^{2}$ Cosmic Garden Co., Ltd., Okayama, Japan; ${ }^{3}$ Specified Non-profit Organisation MEDICAL TECHNO OKAYAMA, Okayama, Japan; ${ }^{4}$ Okayama University Organisation for Research Promotion and Collaboration, Okayama, Japan

\subsection{6/oemed-2018-ICOHabstracts. 166}

Introduction Pollen allergy is major problem in Japan. The custom-homebuilding company, Cosmic Garden Co. Ltd., located in Okayama City, Japan was established in 1997 and uses specific natural ore powder (SNOP) in wall materials a to improve allergic symptoms. In this study, we surveyed biological effects of short term stay in a room surrounded by SNOP cloth.

Methods To investigate the biological effects of SNOP, patients with a pollen allergy were recruited to stay in a room surrounded by cloth containing SNOP (CCSNOP), and their symptoms and various biological parameters were compared with those of individuals staying in a room surrounded by control non-woven cloth (NWC). Each stay lasted $60 \mathrm{~min}$. Before and immediately after the stay, a questionnaire regarding allergic symptoms, as well as POMS (Profile of Mood Status) and blood sampling, was performed. Post-stay minus prestay values were calculated and compared between CCSNOP and NWC groups.

Results Results indicated that some symptoms, such as nasal obstruction and lacrimation, improved, and POMS evaluation showed that patients were calmer following a stay in CCSNOP. Relative eosinophils, non-specific Ig E, epidermal growth factor, monocyte chemotactic protein-1, and tumour necrosis factor- $\alpha$ increased following a stay in CCSNOP.

Conclusion This ore powder improved allergic symptoms, and long-term monitoring involving 1 to 2 months may be necessary to fully explore the biological and physical effects of SNOP on allergic patients.

\section{CLINICAL EVALUATION OF CENP-B AND SCL-70 AUTOANTIBODIES IN SILICOSIS PATIENTS}

${ }^{1} \mathrm{~S}$ Lee ${ }^{*},{ }^{2} \mathrm{H}$ Hayashi, ${ }^{1} \mathrm{~N}$ Kumagai-Takei, ${ }^{1} \mathrm{H}$ Matsuzaki, ${ }^{1} \mathrm{~K}$ Yoshitome, ${ }^{1} \mathrm{Y}$ Nishimura, ${ }^{3} \mathrm{~K}$ Uragami, ${ }^{4} \mathrm{M}$ Kusaka, ${ }^{1} \mathrm{~S}$ Yamamoto, ${ }^{1} \mathrm{M}$ Ikeda, ${ }^{1} \mathrm{~T}$ Hatayama, ${ }^{2} \mathrm{~W}$ Fujimoto, ${ }^{1} \mathrm{~T}$ Otsuki. ${ }^{1}$ Dept. Hygiene, Kawasaki medical school, Kurashiki, Japan; ${ }^{2}$ Dept. Dermatology, Kawasaki medical school, Kurashiki, Japan; ${ }^{3}$ Hinase Uragami lin, Bizen, Okayama, Japan; ${ }^{4}$ Kusaka Hospital, Okayama, Japan

\subsection{6/oemed-2018-ICOHabstracts. 167}

Introduction Silicosis patients (SIL) suffer from respiratory disorders and dysregulation of autoimmunity. Frequent complications such as rheumatoid arthritis, systemic sclerosis (SSc) and vasculitis are known in SIL. Furthermore, we reported previously that some SIL exhibited better respiratory conditions in association with a worse immunological status. In this study, the clinical roles of anti-CENP-B and Scl-70 autoantibodies in SIL were analysed.

Methods Plasma samples were collected from Healthy Volunteers (HV), SIL, Systemic Sclerosis (SSc). Plasma factors and autoantibodies were determined by ELISA. Statistical analysis were performed by SPSS (IBM).

Results The titer index ( $\log 10)$ of anti-CENP-B autoantibody in SIL was higher than that of HV and that of SSc was higher than those of HV and SIL. This titer index was positively correlated with an assumed immune status of 1 for $\mathrm{HV}, 2$ for SIL, and 3 for SSc. Moreover, although factor analysis revealed that the titer index of the anti-CENP-B autoantibody formed the same factor with the anti-Scl-70 autoantibody, IgG value and age in SIL cases, another extracted factor indicated that the $\operatorname{IgA}$ value and anti-Scl-70 antibody were positively related, but anti-CENP-B showed an opposite pattern in the results of the factor analysis.

Conclusion These findings indicated that the titer index of anti-CENP-B autoantibody may be a biomarker for dysregulation in SIL cases. Future clinical follow-up of SIL may therefore require both respiratory and immunological assessment

\section{Cardiology in Occupational Health}

\section{THE ASSESSMENT OF OCCUPATIONAL RISKS OF ACCELERATED AGEING AMONG RUSSIAN PROFESSIONAL DRIVERS}

1,2Bashkireva Angelika*. 'Gerontological Centre of Leningrad Region, St. Petersburg, Russia; ${ }^{2}$ Research and Innovation Centre "Professional Longevity», St. Petersburg, Russia

\subsection{6/oemed-2018-ICOHabstracts. 168}

Introduction We know the phenomenon of differential ageing to be the result of unequal environment conditions pressure. It is necessary to assess the difference between calendar and biological age (BA) in order to reveal the effects induced by combined occupational hazards in professional lorry-drivers. We have compared the physical (PWC) and mental work capacity (MWC), BA and ageing rates of lorry-drivers (experimental group) and labourers (control group) in connexion with their chronological age (CA), driving experience, occupational environment, work schedule and social-demographic characteristics.

Methods 150 male lorry-drivers (mean age $41.3 \pm 0.9$ ) and 150 male labourers (mean age 44.8 \pm 0.9 ) were examined according to the multiply-regressional model of BA evaluation based on 